\title{
A note on the definition of deformed exponential and logarithm functions
}

\author{
Thomas Oikonomou ${ }^{1, a)}$ and G. Baris Bagci ${ }^{2}$ \\ ${ }^{1}$ Centro Brasileiro de Pesquisas Fisicas, Xavier Sigaud 150, 22290-180 Rio de Janeiro, \\ Brazil and Institute of Physical Chemistry, National Center for Scientific Research \\ "Demokritos," 15310 Athens, Greece \\ ${ }^{2}$ Department of Physics, Faculty of Science, Ege University, 35100 Izmir, Turkey
}

(Received 26 June 2009; accepted 20 August 2009; published online 6 October 2009)

\begin{abstract}
The recent generalizations of the Boltzmann-Gibbs statistics mathematically rely on the deformed logarithmic and exponential functions defined through some deformation parameters. In the present work, we investigate whether a deformed logarithmic/exponential map is a bijection from $\mathbb{R}^{+} / \mathbb{R}$ (set of positive real numbers/ all real numbers) to $R / \mathbb{R}^{+}$, as their undeformed counterparts. We show that their inverse map exists only in some subsets of the aforementioned (co)domains. Furthermore, we present conditions which a generalized deformed function has to satisfy, so that the most important properties of the ordinary functions are preserved. The fulfillment of these conditions permits us to determine the validity interval of the deformation parameters. We finally apply our analysis to Tsallis $q$-deformed functions and discuss the interval of concavity of the Rényi entropy. () 2009 American Institute of Physics. [doi:10.1063/1.3227657]
\end{abstract}

\section{INTRODUCTION}

In order to generalize the Boltzmann-Gibbs (BG) entropy and associated statistics, there have been a multitude of entropy definitions such as the Tsallis, ${ }^{1}$ Kaniadakis, ${ }^{2}$ and Rényi ${ }^{3}$ generalized entropies. The central goal of these generalizations is the derivation of nonexponential distributions, observed in a large number of phenomena in various fields of physical sciences involving, among others, long-range correlations, nonergodicity, long-time memories, (multi)fractal structures, and self-similarities. An important thermostatistics in this regard is the one proposed originally by Tsallis ( $q$-statistics) ${ }^{1}$ yielding asymptotically inverse power law distributions. The $q$-statistics has been applied to a vast number of physical situations in various scientific areas, of which a comprehensive review can be found in Ref. 4. Similarly, one can obtain power law distributions in the thermodynamic limit through a different generalization introduced by Kaniadakis $\left(\kappa\right.$-statistics) ${ }^{2}$ developed in the context of special relativity.

Considering trace-form entropies, $\Sigma_{i} \Lambda_{\xi}\left(p_{i}\right)=\Sigma_{i} p_{i}\left(\Lambda_{\xi}\left(p_{i}\right) / p_{i}\right)$, where $\boldsymbol{\xi}=\left\{\xi_{j}\right\}_{j=1, \ldots, u}$ is a deformation parameter set, $p_{i}$ is the probability of the $i$ th-microstate, and $\Lambda_{\xi}\left(p_{i}\right)$ is a probability functional, the generalization procedure consists in introducing deformed logarithmic and exponential functions, ${ }^{5} f_{\xi}: \mathbb{F}^{+} \subseteq \mathbb{R}^{+} \rightarrow \mathbb{F} \subseteq \mathbb{R}$ with $f_{\xi}(x):=x \Lambda_{\xi}(1 / x)$ where $\mathbb{F}\left(\mathbb{F}^{+}\right)$is a subset of all (positive) real numbers. Similarly, the deformed inverse function $f_{\xi}^{-1}$ is a mapping, namely, $f_{\xi}^{-1}: \mathbb{F} \subseteq \mathbb{R}$ $\rightarrow \mathbb{F}^{+} \subseteq \mathbb{R}^{+}$with $f_{\xi}^{-1}(x):=$ Inverse $\left\{x \Lambda_{\xi}(1 / x)\right\}$. These deformed functions are required to recover the ordinary definitions for specific (and unique) values $\xi_{0}$ of the deformation parameters, i.e., $f_{\xi_{0}}(x)=\ln (x)$ and $f_{\xi_{0}}^{-1}(x)=\exp (x)$. The aforementioned $\boldsymbol{\xi}$-generalized functions usually present mathematical properties which differ from the ordinary, undeformed ones. For example, we generally observe for the deformed logarithms

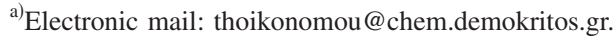




$$
f_{\xi}(x y) \neq f_{\bar{\xi}}(x)+f_{\xi}(y)
$$

when $\boldsymbol{\xi} \neq \boldsymbol{\xi}_{0}$. Equivalently, assuming the existence of the inverse function $f_{\boldsymbol{\xi}}^{-1}(x)$, the equation above can be written in terms of the deformed exponentials as

$$
f_{\xi}^{-1}(x+y) \neq f_{\xi}^{-1}(x) f_{\xi}^{-1}(y) .
$$

These two equations exhibit the underlying mathematical structure of the generalized exponentials and their inverses, namely, the generalized logarithms. Evidently, the introduction of the deformation parameter $\boldsymbol{\xi}$ serves as a coupling between the variables $x$ and $y$.

In this paper, we first show that the deformed functions are generally not invertible in the entire (co)domains of the ordinary functions as a consequence of the aforementioned inequalities. In this sense, the deformed functions used so far are found to be incomplete. Then, we present the conditions under which bijectivity is satisfied. Incorporating these conditions, we define complete generalized logarithms and exponentials. Finally, we apply the criterion of completeness to Tsallis generalized functions in order to elucidate some obscure points in diverse mathematical constructions within the frame of generalized BG statistics. As a result of our study, it becomes evident that the justification of whether a function can be considered as generalized logarithmic or generalized exponential function is associated with some additional properties than just a recovering limit $\boldsymbol{\xi} \rightarrow \boldsymbol{\xi}_{0}$.

The paper is organized as follows. In Sec. II we present the origin of the nonbijectivity of the $\xi$-logarithm (-exponential) from $\mathbb{R}^{+}(\mathbb{R})$ to $\mathbb{R}\left(\mathbb{R}^{+}\right)$. In order to assure the invertibility of the $\xi$-functions in the aforementioned sets, we introduce a dependence between deformation parameters and specific subsets $\mathrm{L}_{i}$ and $\mathbb{K}_{i}$ of $\mathbb{R}^{+}\left(\mathbb{R}^{+}=\cup_{i=1}^{n=2} \mathrm{~L}_{i}\right)$ and $\mathbb{R}\left(\mathbb{R}=\cup_{i=1}^{n=2} \mathrm{~K}_{i}\right)$, respectively. Through the requirement of bijectivity, we are able to determine explicitly the subsets $\mathrm{L}_{i}$ and $K_{i}$. In addition, we set forth conditions which permit us the determination of the respective parameter validity ranges. In Sec. III, we apply our formalism to $q$-deformed functions of Tsallis thermostatistics and discuss the interval of concavity of the Rényi entropy. Finally, the main results are summarized in Sec. IV.

\section{DEFINITION INTERVALS OF DEFORMED FUNCTIONS}

We first define the following intervals $\mathbb{L}_{0}:=\{x \in \mathbb{R}: 0<x \leq 1\}$ and $\mathbb{L}_{1}:=\{x \in \mathbb{R}: x \geq 1\}$ so that $\mathbb{L}_{0} \cup \mathbb{L}_{1}=\mathbb{R}^{+}:=\{x \in \mathbb{R}: x>0\}$, i.e., the set of all positive real numbers. We also choose the intervals $\mathbb{K}_{1}$ and $\mathbb{K}_{2}$ as $\mathbb{R}_{0}^{+}:=\{x \in \mathbb{R}: x \geq 0\}$ and $\mathbb{R}_{0}^{-}:=\{x \in \mathbb{R}: x \leq 0\}$, respectively, so that $\mathbb{R}=\mathbb{R}_{0}^{-} \cup \mathbb{R}_{0}^{+}$is the set of all real numbers. We denote the parameter validity ranges of the respective parameters $\boldsymbol{\xi}$ by $\mathcal{A}_{\xi} \subseteq \mathbb{R}$. A boundary value of $\mathcal{A}_{\boldsymbol{\xi}}$ is always $\boldsymbol{\xi}_{0}$, e.g., $\mathcal{A}_{\xi}:=\left(\alpha, \boldsymbol{\xi}_{0}\right]$ with $\alpha<\boldsymbol{\xi}_{0}$ or $\mathcal{A}_{\xi}:=\left[\boldsymbol{\xi}_{0}, \alpha\right)$ with

$\alpha>\xi_{0}$. For the sake of simplicity, we shall conduct our study starting from Eq. (1), although the same results can analogously be obtained from Eq. (2).

The ordinary logarithm (in natural base $e$ ) $\ln : \mathbb{R}^{+} \rightarrow \mathbb{R}$ has an argument intercept at the point $x=1$, since $\ln (x=1)=0$, splitting the logarithmic domain $\mathbb{R}^{+}$into two subdomains $\mathbb{L}_{0}$ and $\mathrm{L}_{1}$. The relation between the images of the log arguments $x \in \mathbb{L}_{1}$ and $1 / x \in \mathbb{L}_{0}$ is represented by the identity

$$
\ln (x)+\ln (1 / x)=0 \quad\left(f_{\xi_{0}}(x)+f_{\xi_{0}}(1 / x)=0\right) .
$$

The right hand side of Eq. (3), being equal to zero, guarantees that for $x \in \mathbb{R}^{+}$, the logarithmic codomain is $\mathbb{R}$. This can be easily verified if we assume that the sum of the above logarithmic terms does not cancel the $x$-dependence i.e., $\ln (x)=-\ln (1 / x)+\Theta(x)$, where $\Theta$ is a real nonsingular function. Then, the codomain of the logarithmic function depends on the codomain of $\Theta(x)$ as well; thus $\ln : \mathbb{R}^{+} \rightarrow \mathrm{M} \subseteq \mathbb{R}$ where $\mathbb{M}$ denotes any subset of real numbers.

We now require the same (co)domain for $f_{\xi}$ as that of $f_{\xi_{0}}$ and an analogous behavior of its image, namely, $f_{\xi}: \mathbb{L}_{0} \cup \mathbb{L}_{1} \rightarrow \mathbb{R}_{0}^{-} \cup \mathbb{R}_{0}^{+}, f_{\xi}\left(x \in \mathbb{L}_{0}\right) \in \mathbb{R}_{0}^{-}$and $f_{\xi}\left(x \in \mathbb{L}_{1}\right) \in \mathbb{R}_{0}^{+}$. Setting $y \rightarrow 1 / x$ in Eq. (1), we are able to explore the relation between the $x$ - and $1 / x$-arguments of $f_{\xi}$. Then, for 
$\boldsymbol{\xi} \neq \boldsymbol{\xi}_{0}$, we observe that $f_{\boldsymbol{\xi}}(x)+f_{\xi}(1 / x) \neq f_{\xi}(1)$. Since the term $f_{\xi}(1)$ is a constant with respect to the $x$-variable and may take any value in $\mathbb{R}$, the latter relation implies the existence of an $x$-dependent function on the right hand side unlike the ordinary case i.e.,

$$
f_{\xi}(x)+f_{\xi}(1 / x)=\Theta_{\xi}(x) .
$$

Then, the generalized $\operatorname{logarithm} f_{\xi}$ represents a possible generalization only in a subset of the codomain of the ordinary logarithmic function. In other words, it is incomplete compared to its ordinary counterpart. Therefore, we call a deformed function which is characterized by Eq. (4) with $\Theta_{\xi}(x) \neq 0$ an incomplete generalized logarithm, $f_{\xi}: \mathbb{R}^{+} \rightarrow \mathrm{M}_{\xi} \subseteq \mathbb{R}$, with its inverse being incomplete generalized exponential function i.e., $f_{\xi}^{-1}: \mathbb{M}_{\xi} \subseteq \mathbb{R} \rightarrow \mathbb{R}^{+}$. An incomplete definition has three major setbacks: First, as mentioned above, the codomain $\mathbb{M}_{\xi}$ is a subset of $\mathbb{R}$. This is not a desired property, since it may restrict the range of the argument interval in the $\xi$-exponential $f_{\xi}^{-1}$. It is always possible that a particular physical system demands the use of an argument external to the particular subset $\mathrm{M}_{\xi}$. If this is the case, then the applicability of the statistics based on incomplete deformed functions will be impossible in the presumed interval as a result of the underlying mathematical structure rather than the requirements of the physical system under consideration. Second, the subset $\mathbb{M}_{\xi}$ depends on the deformation parameters, providing a further restriction in the domain of $f_{\xi}^{-1}$. In other words, the applicability of the generalized statistics is limited to a certain range depending on the deformation parameter right from the beginning independent of the physical system of interest. Therefore, it is ambiguous whether the resulting deformation parameter range is a result of the physical model or just chosen from the accessible range due to the nature of the deformation parameter. The third setback is that the function $f_{\xi}^{-1}(x)$ does not describe a $\boldsymbol{\xi}$-exponential decay for $x<0$ due to the dependence of $\mathrm{M}_{\xi}$ on the deformation parameters. This can be observed by inverting Eq. (4), which yields the relation $f_{\xi}^{-1}(-x)$ $=1 / f_{\xi}^{-1}\left(x+\Theta_{\xi}^{\prime}(x)\right)$, where $\Theta_{\xi}^{\prime}(x):=\Theta_{\xi}\left(1 / f_{\xi}^{-1}(-x)\right)$. This relation implies that $f_{\xi}^{-1}(x)$ represents a $\xi$-exponential decay only when $\Theta_{\xi}^{\prime}(x)=0$. The aforementioned observation can also be made by recourse to Eq. (2), since it yields the relation $f_{\xi}^{-1}(-x) f_{\xi}^{-1}(x) \neq f_{\xi}^{-1}(0)$ if we set $y \rightarrow-x$.

An immediate question then is whether one can define a complete generalized logarithm. This consists of two steps: (i) to eliminate the $\boldsymbol{\xi}$-dependence of the codomain, $\mathbb{M}_{\xi} \rightarrow \mathbb{M}$ and (ii) to increase the range of the codomain, such that $\mathrm{M}=\mathbb{R}$. Since the origin of the incompleteness lies in the existence of nonzero $\Theta_{\xi}(x)$ in Eq. (4), the elimination of the latter function would satisfy the aforementioned steps simultaneously. In order to do this, we assume the existence of the functions $d_{k}: \mathcal{A}_{\xi} \rightarrow \mathcal{B}_{\xi}^{(k)}\left(\mathcal{B}_{\xi}^{(k)} \subseteq \mathbb{R}\right)$ with $k=1, \ldots, v \geq u$ such that

$$
f_{\xi}(x)+f_{d_{k}(\xi)}(1 / x)=f_{\xi}(1),
$$

with $\lim _{\xi \rightarrow \xi_{0}} d_{k}(\xi)=\xi_{0}$. The index $k$ associates a $d$-function with each parameter $\xi_{j}, v=u$. However, it is possible to express the $i$ th deformation parameter as a function of the $\ell$ th one $(j=1, \ldots, i, \ldots, \ell, \ldots, u), \xi_{i}=\xi_{i}\left(\xi_{\ell}\right)$, so that we have two different deformation parametric structures based on the same deformation parameter. This degeneracy is captured when $v>u$. Setting $x=1$ in Eq. (5), we obtain $f_{d_{k}(\xi)}(1)=0$ in accordance with the ordinary logarithm. It can be seen that the above relation is valid for any set of parameters by substituting $\boldsymbol{\xi}^{\prime}=d_{k}(\boldsymbol{\xi})$ into the above equation. Therefore, Eq. (5) can be rewritten as

$$
f_{\xi}(x)=-f_{d_{k}(\xi)}(1 / x),
$$

in analogy to Eq. (3). It is worth remarking that one can obtain Eq. (6) by changing the term $f_{\xi}(1)$ in Eq. (5) to $f_{d_{k}(\xi)}(1)$, as well. Because of Eq. (4), we have $d_{k}(\xi) \neq \xi$, which means that even if the domain $\mathcal{A}_{\xi}$ is equal to the codomain $\mathcal{B}_{\xi}^{(k)}$, the image of $d_{k}(\xi)$ (except at the boundary value $\boldsymbol{\xi}_{0}$ ) is not equal to the image of $\boldsymbol{\xi}$. We denote $d$ as dual function and the correspondence $\boldsymbol{\xi} \leftrightarrow d_{k}(\boldsymbol{\xi})$ as duality relation. The explicit structure of the dual mapping depends on the particular expression of the generalized functions. 
It should also be noted that the transformation of the arguments, i.e., $x \rightarrow 1 / x$ given by Eq. (6), keeping the deformation parameters invariant, can equivalently be considered as the transformation of the parameters as well, $\boldsymbol{\xi} \rightarrow \widetilde{\boldsymbol{\xi}}$ and $d_{k}(\boldsymbol{\xi}) \rightarrow \widetilde{d}_{k}(\widetilde{\boldsymbol{\xi}})$, yielding the expression

$$
f_{\tilde{d}_{k}}(\tilde{\xi})(x)=-f_{\tilde{\xi}}(1 / x),
$$

with $\boldsymbol{\xi} \neq \widetilde{\boldsymbol{\xi}}$. The function $\widetilde{d}_{k}$ has the same expression with $d_{k}$, since the dual function is unique for each $\boldsymbol{\xi}$-function, but presents the reverse (co)domain, $\widetilde{d}_{k}: \mathcal{B}_{\tilde{\xi}}^{(k)} \rightarrow \mathcal{A}_{\tilde{\xi}}$, with $\mathcal{A}_{\xi}=\mathcal{A}_{\tilde{\xi}}$ and $\mathcal{B}_{\xi}^{(k)}=\mathcal{B}_{\tilde{\xi}}^{(k)}$. We further observe that the argument $x=1$, since $f_{\xi}(1)=0$, divides the (co)domain $\mathbb{R}^{+}(\mathbb{R})$ into the $\operatorname{sub}$ (co)domains $\mathbb{R}^{+}=\mathbb{L}_{0} \cup \mathbb{L}_{1} \quad\left(\mathbb{R}=\mathbb{R}^{-} \cup R_{0}^{+}\right)$. Consequently, we are led to the complete $\xi$-logarithmic definition, $\ln _{\xi}$, from $\mathbb{R}^{+}$to $\mathbb{R}$ with $\xi \in \mathcal{A}_{\xi}$ when the subsets of the (co)domain correspond to different deformation parameters, i.e.,

$$
\ln _{\xi}:=\left\{\begin{array}{l}
f_{d_{k}(\xi)}: \mathbb{L}_{0} \rightarrow \mathbb{R}_{0}^{-} \\
f_{\xi}: L_{1} \rightarrow \mathbb{R}_{0}^{+}
\end{array} \quad \text { with } \quad \exp _{\xi} \equiv \ln _{\xi}^{-1}:=\left\{\begin{array}{l}
f_{d_{k}(\xi)}^{-1}: \mathbb{R}_{0}^{-} \rightarrow \mathbb{L}_{0} \\
f_{\xi}^{-1}: \mathbb{R}_{0}^{+} \rightarrow \mathbb{L}_{1}
\end{array} .\right.\right.
$$

We can then, for the complete generalized logarithm and exponential given above, verify that

$$
\begin{aligned}
& \ln _{\xi}(1 / x)=f_{d_{k}(\xi)}(1 / x)=-f_{\xi}(x)=-\ln _{\xi}(x), \\
& \exp _{\xi}(-x)=f_{d_{k}(\xi)}^{-1}(-x)=\frac{1}{f_{\xi}^{-1}(x)}=\frac{1}{\exp _{\xi}(x)}
\end{aligned}
$$

for $x \in \mathbb{L}_{1}$ and $x \in \mathbb{R}_{0}^{+}$, respectively. On the other hand, when a deformed logarithm fulfills the relation in Eq. (4) with $\Theta_{\xi}(x)=0$, the transformation $x \rightarrow 1 / x$ does not cause any structural changes, implying that $f_{\boldsymbol{\xi}}$ is $\boldsymbol{\xi}$-symmetric, i.e., $f_{\boldsymbol{\xi}}$ and $f_{d_{k}(\boldsymbol{\xi})}$ have the same images. Combining Eqs. (4) and (6), we are able to construct a criterion based on the dual function $d_{k}(\xi)$ and the primary deformed function $f_{\xi}$. A deformed function $f_{\xi}$ represents a single-parameter-set complete and analytic generalized logarithm, $f_{\xi}: \mathbb{R}^{+} \rightarrow \mathbb{R}\left(f_{\xi} \equiv \ln _{\xi}\right)$, when the following relation is satisfied:

$$
f_{d_{k}(\xi)}(x)=f_{\xi}(x) \text { for } \quad d_{k}(\xi) \neq \boldsymbol{\xi} .
$$

The same criterion can be expressed for the inverse deformed function $f_{\xi}^{-1}$ through the relation $f_{\xi}^{-1}(-x) f_{d_{k}(\xi)}^{-1}(x)=1$.

The parameter range $\mathcal{A}_{\xi}$ can be determined by requiring the fulfillment of the following limits satisfied by the ordinary functions:

$$
\begin{gathered}
\lim _{x \rightarrow 0} \exp _{\xi}(x)=1, \quad \lim _{x \rightarrow 1} \ln _{\xi}(x)=0, \\
\lim _{x \rightarrow-\infty} \exp _{\xi}(x)=0, \quad \lim _{x \rightarrow 0} \ln _{\xi}(x)=-\infty, \\
\lim _{x \rightarrow \infty} \exp _{\xi}(x)=\infty, \quad \lim _{x \rightarrow \infty} \ln _{\xi}(x)=\infty, \\
\lim _{x \rightarrow-\infty} \frac{d}{d x} \exp _{\xi}(x)=0, \quad \lim _{x \rightarrow \infty} \frac{d}{d x} \ln _{\xi}(x)=0 .
\end{gathered}
$$

Condition (10a) implies that $\ln _{\xi}(x)$ and $\exp _{\xi}(x)$ are continuous functions at the points $x=1$ and $x=0$, respectively. Equations (10b) and (10c) describe the behavior of the generalized functions at the boundaries of their domains. Condition (10d) ensures the preservation of the same absolute 
maximum and minimum of the ordinary functions. As we shall see in Sec. III, the conditions listed in Eq. (10) are in complete agreement with the definitions in Eq. (8), yielding a complete definition of the generalized functions.

\section{TSALLIS $q$-FUNCTIONS}

Having introduced the criterion of the completeness in the context of the generalized functions, we now apply it to the Tsallis generalized functions in order to obtain concomitant complete expressions. Moreover, we will show that the criterion of completeness naturally yields the concavity interval of the Rényi entropy if one defines the Rényi entropy through $q$-deformed generalized functions and thereby using the completeness of these functions.

A one parameter $\left(\boldsymbol{\xi}=\left\{\xi_{1}\right\}=: q\right.$ with $\left.q_{0}=1\right)$ generalization introduced by Tsallis ${ }^{1,4}$ reads

$$
f_{q}(x):=\frac{x^{1-q}-1}{1-q}, \quad h_{q}(x):=[1+(1-q) x]^{1 /(1-q)},
$$

with $f_{q \rightarrow 1}(x)=\ln (x)$ and $h_{q \rightarrow 1}(x)=\exp (x)$. It can be easily shown that $f_{q}(x) \neq-f_{q}(1 / x)$, since $\Theta_{q}(x)=\left(x^{1-q}+x^{q-1}-2\right) /(1-q) \neq 0$ [or equivalently $h_{q}(x) h_{q}(-x) \neq 1$ where $h_{q}(x) \equiv f_{q}^{-1}(x)$ ]. Concerning their domain and codomain, we observe

$$
\begin{aligned}
& q<1 \quad f_{q}: \mathbb{R}_{0}^{+} \rightarrow\left[\frac{1}{q-1}, \infty\right), \quad h_{q}:\left[\frac{1}{q-1}, \infty\right) \rightarrow \mathbb{R}_{0}^{+}, \\
& q=1 \quad f_{q}: \mathbb{R}^{+} \rightarrow \mathbb{R}, \quad h_{q}: \mathbb{R} \rightarrow \mathbb{R}^{+}, \\
& q>1 \quad f_{q}: \mathbb{R}^{+} \rightarrow\left(-\infty, \frac{1}{q-1}\right], \quad h_{q}:\left(-\infty, \frac{1}{q-1}\right] \rightarrow \mathbb{R}^{+} .
\end{aligned}
$$

The generalized functions, $f_{q}$ and $h_{q}$, are not complete when $q \neq 1$ as can be seen from Eq. (12). This limits the use of deformed functions given by (11) to some subsets of the real numbers for $q \neq 1$, not as a result of the physical system under investigation but solely due to the incompleteness of the underlying mathematical structure. Since one first needs the dual function $d_{k=1}(q)=d(q)$ in order to define complete generalized functions, we calculate it from Eqs. (6) and (11) as

$$
d(q)=2-q
$$

We notice that the analytical expression in Eq. (13) depends as much on $q$ as on the recovering value $q_{0}$. For example, the reparametrization $q^{\prime}=1-q$ in the above equation changes the dual mapping $d(q)$ to $d^{\prime}\left(q^{\prime}\right)=-q^{\prime}$ with $q_{0}^{\prime}=0$. The requirements listed in Eq. (10) confine the values of the deformation parameter $q$ into the following interval:

$$
\mathcal{A}_{q}:=(0,1]
$$

Note that $\mathcal{A}_{q}$ coincides with the parameter interval obtained from the normalization of the $q$-exponential decay, i.e., $\int_{0}^{\infty} d x / h_{q}(x)=1$.

Having explicitly obtained the dual mapping function $d(q)$ and the range of validity of the deformation parameter $q$, we can now define the complete $q$-generalized functions, 


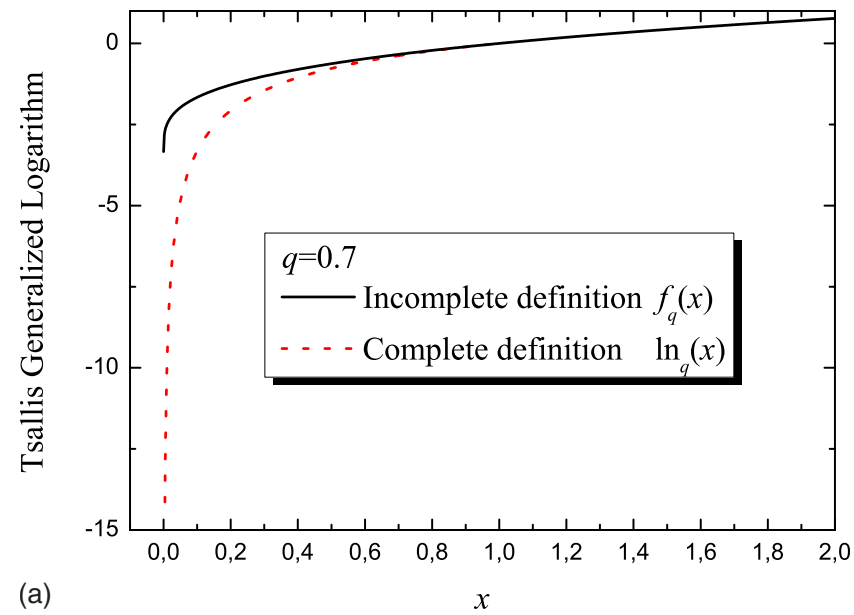

(a)

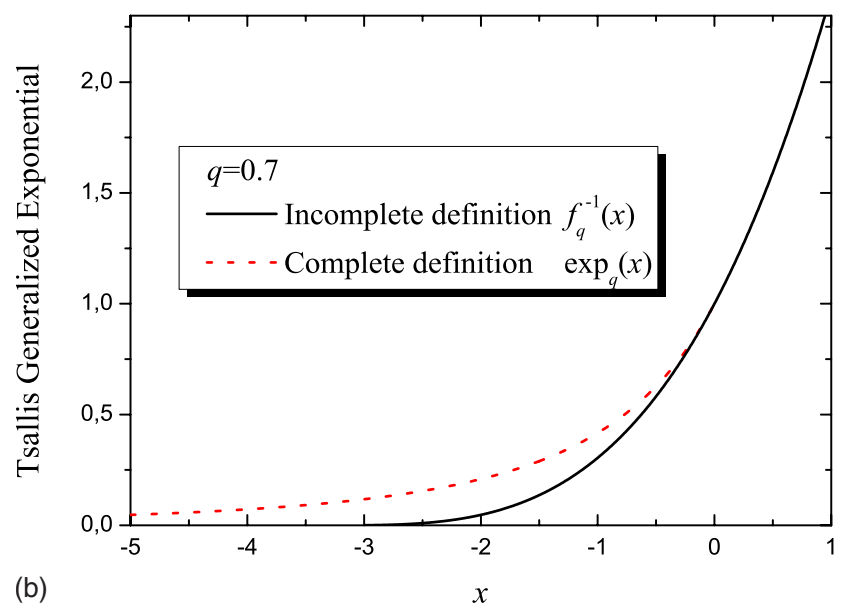

FIG. 1. (Color online) (a) Plot of the Tsallis incomplete $\left(f_{q}\right.$, solid line) and complete ( $\ln _{q}$, dotted line) deformed logarithms for $q=0.7$. (b) Plot of the Tsallis incomplete $\left(f_{q}^{-1}\right.$, solid line) and complete ( $\exp _{q}$, dotted line) deformed exponentials for $q=0.7$.

$$
\ln _{q}(x):=\left\{\begin{array}{ll}
\frac{x^{q-1}-1}{q-1}, & x \in \mathbb{L}_{0}, \\
\frac{x^{1-q}-1}{1-q}, & x \in \mathbb{L}_{1},
\end{array} \quad \exp _{q}(x):= \begin{cases}{[1+(q-1) x]^{1 /(q-1)},} & x \in \mathbb{R}_{0}^{-}, \\
{[1+(1-q) x]^{1 /(1-q)},} & x \in \mathbb{R}_{0}^{+},\end{cases}\right.
$$

in accordance with Eq. (8). These expressions are also obtained, with a slightly different choice, by Teweldeberhan et al. in Ref. 6 in the context of cutoff prescriptions associated with the $q$-generalized functions. These authors too have noticed that a division of the (co)domains yields to a complete definition of the $q$-exponentials. However, this observation has not been pursued further as a criterion of completeness in Ref. 6.

We plot the incomplete (solid lines) and complete (dashed lines) $q$-generalized logarithms in Eqs. (11) and (15), respectively, corresponding to the deformation parameter $q=0.7$, in Fig. 1(a). The incomplete $q$-logarithm $f_{q}$ has a cutoff at the point $x=0$ corresponding to the value $1 /(q-1)$, which has no analog in the ordinary logarithmic function. The complete $q$-logarithm $\ln _{q}$, on the other hand, behaves as the ordinary logarithmic function, tending to $\ln _{q}(x) \rightarrow-\infty$ as $x \rightarrow 0$. The complete and incomplete $q$-logarithms exhibit the same behavior for $x \geq 1$ as expected, since they are defined from the same domain to the same codomain. For the interval $x<1$, the incomplete generalized logarithm reaches the cutoff and stabilizes therein, whereas the complete 
$q$-logarithm, mapped by the dual function, smoothly approaches $-\infty$. An inspection of Fig. 1(b) reveals the analogous results for the incomplete and complete generalized exponentials for $q$ $=0.7$.

\section{A. Rényi entropy}

One of the most discussed deformed entropic structures introduced first within information theory and then as a possible generalization of the BG thermostatistics is the one defined by Rényi, ${ }^{3,7}$

$$
S_{r}=\frac{1}{1-r} \ln \left(\sum_{i} p_{i}^{r}\right)
$$

where $r$ is the deformation parameter and $S_{r \rightarrow 1}=S_{\mathrm{BG}}$. It has been previously shown that it exhibits a well-defined concavity only for $r \in(0,1) .{ }^{8}$ The nonconcavity of the Rényi entropy outside the aforementioned range has been frequently used as an argument for the inappropriateness of the $S_{r}$-definition as a candidate for the BG generalization.

The BG entropy can be derived, without appealing to physical laws, by considering the asymptotic behavior of the ordinary multinomial coefficients (OMCs). The OMC gives the number of all possible configurations - or (micro)states in physics - created from the combination of objects without repetitions. In a recent study, ${ }^{9}$ one of the present authors (T.O.) constructed properly defined deformed multinomial coefficients (DMCs), based on the concept of the deformation parameters, in order to derive the Tsallis, Rényi, and nonextensive Gaussian ${ }^{10}$ entropies. Demanding the positivity of DMC, since a negative number of configuration is senseless, it has been demonstrated that the Rényi entropy is defined only for $r \in(0,1)$. The same result about the parameter validity range is obtained in Ref. 11 by applying Jaynes' formalism for $S_{r}$ with ordinary (in contrast to the escort) exponentially averaged constraints in accordance with the fundamental mathematical structure of the Rényi entropy.

We now derive the interval of concavity in a more fundamental way following a different path, related to the parameter range in Eq. (14) and the definitions given by Eq. (15). In Ref. 9, it has been established that the Rényi entropy can be expressed through the deformed multiplication introduced in Ref. 12 within the Tsallis statistics $\left[x \otimes_{r} y:=\exp _{r}\left(\ln _{r}(x)+\ln _{r}(y)\right), x, y>1\right]$ as follows:

$$
S_{r}=\ln \left(\prod_{i} \otimes_{r}\left(1 / p_{i}\right)^{\otimes_{r}^{p_{i}}}\right), \quad \prod_{i} \otimes_{r}\left(1 / p_{i}\right)^{\otimes_{r}^{p_{i}}}=\exp _{r}\left(\sum_{i} p_{i} \ln _{r}\left(1 / p_{i}\right)\right)=\left[\sum_{i} p_{i}^{r}\right]^{1 /(1-r)} .
$$

Assuming that the deformed functions in Eq. (17) are complete, we see that the $\ln _{r}$-argument takes values in $\mathbb{L}_{1}$ (i.e., the microprobabilities vary between zero and one), and thus the $\exp _{r}$-argument varies in the interval $\mathbb{R}_{0}^{+}$. It becomes then evident that the deformation parameter $r$ takes values in $(0,1)$ as a consequence of the mathematical definition of the generalized functions in Eq. (15). Accordingly, the Rényi entropy is concave for all values of its parameter range when the complete deformed functions are being used instead of the incomplete ones. The very fact that one uses the complete generalized functions restricts the parameter range only to values in the interval $(0,1)$. Once we use the complete deformed functions rather than the incomplete ones, the interval of the concavity coincides with the interval of the parameter range.

\section{CONCLUSIONS}

The recent generalizations of the BG statistics are based on the introduction of some deformed forms of the ordinary logarithmic and exponential functions, where $\boldsymbol{\xi}=\left\{\xi_{i}\right\}_{i=1, \ldots, u}$ denotes the deformation parameter set. It is hence of great importance to understand the mathematical structure of these deformed functions in order to gain more insight into the associated generalization schemes. Motivated by this fact, we first demonstrated that the generalized $\operatorname{logarithms} f_{\xi}$ (and their inverse, i.e., generalized exponentials $h_{\xi}$ ) are nonbijective from $\mathbb{R}^{+}(\mathbb{R})$ to $\mathbb{R}\left(\mathbb{R}^{+}\right)$. Thus, the 
generalized logarithm definitions $f_{\xi}$ may represent the inversion of the generalized exponentials $h_{\xi}$ and vice versa only in some subsets of $\mathbb{R}^{+}$and $\mathbb{R}$. Therefore, they are called incomplete deformed functions. This feature issues from the nonadditive $\boldsymbol{\xi}$-logarithmic and nonmultiplicative $\xi$-exponential composition rules, i.e., $f_{\xi}(x y) \neq f_{\xi}(x)+f_{\xi}(y)$ and $h_{\xi}(x+y) \neq h_{\xi}(x) h_{\xi}(y)$. The incompleteness of deformed functions is not only a mathematical deficiency but also presents some physical problems, since it restricts the applicability of the associated thermostatistics. In other words, it is the incomplete mathematical structure which dictates the range of arguments or results rather than the physical system one studies. However, the estimation of the aforementioned subsets is not a trivial procedure, since some boundary values present $x(\xi)$-dependence. In order to overcome this difficulty and to guarantee the property of bijectivity in the original (co)domains, we proposed dual mapping functions $d_{k}(\xi)(k=1, \ldots, v \geq u)$, which enable a change of the parameters within specific subsets of $\mathbb{R}^{+}$and $R$. Through the introduction of such dual functions, one is able to obtain bijective deformed functions, $\ln _{\xi}$ and $\exp _{\xi}$, which are called complete. If $\mathcal{A}_{\xi}, \mathcal{B}_{\xi}^{(k)} \subseteq \mathbb{R}$ are parameter ranges related over a function $d_{k}: \mathcal{A}_{\xi} \rightarrow \mathcal{B}_{\xi}^{(k)}$ such that $f_{\xi}(x)=$ $-f_{d_{k}(\xi)}(1 / x)$ and $h_{\xi}(x)=1 / h_{d_{k}(\xi)}(-x)$, then for $\boldsymbol{\xi} \in \mathcal{A}_{\xi}\left(d_{k}(\xi) \in \mathcal{B}_{\xi}^{(k)}\right)$, the complete deformed functions are defined in the following domains and codomains:

$$
\ln _{\xi}:\left\{f_{d_{k}(\xi)}(x), x \in(0,1]\right\} \cup\left\{f_{\xi}(x), x \in[1, \infty)\right\} \rightarrow\left\{f_{d_{k}(\xi)}(x) \in(-\infty, 0]\right\} \cup\left\{f_{\xi}(x) \in[0, \infty)\right\}
$$

and

$$
\exp _{\xi}:\left\{h_{d_{k}(\xi)}(x), x \in(-\infty, 0]\right\} \cup\left\{h_{\xi}(x), x \in[0, \infty)\right\} \rightarrow\left\{h_{d_{k}(\xi)}(x) \in(0,1]\right\} \cup\left\{h_{\xi}(x) \in[1, \infty)\right\},
$$

with $h_{\xi} \equiv f_{\xi}^{-1}$ and $h_{d_{k}(\xi)} \equiv f_{d_{k}(\xi)}^{-1}$. Further, we posited conditions that the generalized logarithmic and exponential functions have to fulfill in order to preserve the main properties of their ordinary counterparts, e.g., the behavior at the boundary points and the points of extrema. Through these conditions, we determine the ranges $\mathcal{A}_{\xi}$ and $\mathcal{B}_{\xi}^{(k)}$.

Concerning the dual functions $d_{k}(\xi)$, we formulated a criterion to distinguish between deformed logarithmic/exponential structures which are bijective in the original (co)domains, preserving the same parameter for all values of the argument, and those whose bijectivity is assured in the intervals presented above.

The application of our theoretical formalism to Tsallis $q$-definitions led to the parameter ranges $\mathcal{A}_{q}=(0,1]$ and $\mathcal{B}_{q}^{(1)}=[1,2)$ connected to one another through the dual function $d(q)=2-q$. Moreover, writing the Rényi entropy in terms of Tsallis deformed functions, we have obtained the interval of concavity associated with the Rényi entropy by the estimation of the parameter range resulting from the criterion of completeness.

It is worth noting that the criterion of completeness developed here is not an alternative to the generalized sum or product rules. Although these generalized algebras may help preserve some properties of the ordinary exponential and logarithmic functions, they are nevertheless defined in terms of generalized functions. Therefore, they too suffer from the incompleteness of the deformed functions. Moreover, the criterion of completeness is far more general than the approach of generalized algebras, since the latter may drastically change from one generalization to the other. The criterion of completeness, on the other hand, is always based on the same procedure, i.e., calculating the dual functions and the valid parameter intervals.

The current results unveil new perspectives on the consideration of generalized logarithmic and exponential functions, thereby shedding light on various mathematical aspects in need of revision and proper treatment.

\section{ACKNOWLEDGMENTS}

T.O. acknowledges fruitful remarks from E. M. F. Curado, C. Tsallis, R. S. Wedemann, and L. Lacasa. We thank U. Tirnakli for a careful reading of the manuscript and bringing Ref. 6 to our attention. G.B.B. was supported by TUBITAK (Turkish Agency) under Research Project No. 
108T013. T.O. was supported by CNPq (Brazilian Agency) under Research Project No. 505453/ 2008-8.

${ }^{1}$ C. Tsallis, J. Stat. Phys. 521, 479 (1988).

${ }^{2}$ G. Kaniadakis, Phys. Rev. E 66, 056125 (2002).

${ }^{3}$ A. Rényi, Probability Theory (North-Holland, Amsterdam, 1970).

${ }^{4}$ C. Tsallis, Introduction to Onextensive Statistical Mechanics: Approaching a Complex World (Springer, New York, 2009).

${ }^{5}$ Th. Oikonomou and U. Tirnakli, Chaos, Solitons Fractals 42, 3027 (2009).

${ }^{6}$ A. M. Teweldeberhan, A. R. Plastino, and H. G. Miller, Phys. Lett. A 343, 71 (2005).

${ }^{7}$ E. K. Lenzi, R. S. Mendes, and L. R. da Silva, Physica A 280, 337 (2000).

${ }^{8}$ J. D. Ramshaw, Phys. Lett. A 198, 119 (1995); C. Tsallis, ibid. 206, 389 (1995); G. R. Guerberoff and G. A. Raggio, ibid. 214, 313 (1996).

${ }^{9}$ Th. Oikonomou, Physica A 386, 119 (2007).

${ }^{10}$ Th. Oikonomou, Physica A 381, 155 (2007).

${ }^{11}$ G. B. Bagci and U. Tirnakli, Phys. Lett. A 373, 3230 (2009).

${ }^{12}$ E. P. Borges, Physica A 340, 95 (2004); L. Nivanen, A. Le Méhauté, and Q. A. Wang, Rep. Math. Phys. 52, 437 (2003). 